\title{
Quasi-Periodic Solutions of Coupled KDV Type Equations
}

Bo Xue, Fang Li, Xianguo Geng

To cite this article: Bo Xue, Fang Li, Xianguo Geng (2013) Quasi-Periodic Solutions of Coupled KDV Type Equations, Journal of Nonlinear Mathematical Physics 20:1, 61-77, DOI: https://doi.org/10.1080/14029251.2013.792472

To link to this article: https://doi.org/10.1080/14029251.2013.792472

Published online: 04 January 2021 


\title{
Quasi-Periodic Solutions of Coupled KDV Type Equations
}

\author{
Bo Xue* \\ Department of Mathematics, ZhengZhou University, 100 Kexue Road, \\ ZhengZhou, Henan 450001, People's Republic of China \\ xuebo@zzu.edu.cn \\ Fang Li \\ College of Science, Henan University of Technology, Lianhua Road, \\ Zhengzhou, Henan 450001, People's Republic of China \\ lfseafish@163.com \\ Xianguo Geng \\ Department of Mathematics, ZhengZhou University, 100 Kexue Road, \\ ZhengZhou, Henan 450001, People's Republic of China \\ xggeng@zzu.edu.cn \\ Received 2 October 2012 \\ Accepted 5 November 2012
}

\begin{abstract}
A hierarchy of new nonlinear evolution equations is proposed, which are composed of the positive and negative coupled KdV flows. Based on the theory of algebraic curve, the corresponding flows are straightened under the Abel-Jacobi coordinates. The meromorphic function $\phi$, the Baker-Akhiezer vector $\bar{\psi}$, and the hyperelliptic curve $\mathscr{K}_{n}$ are introduced by which quasi-periodic solutions of the first two nonlinear evolution equations in the hierarchy are constructed according to the asymptotic properties and the algebro-geometric characters of $\phi, \bar{\psi}$ and $\mathscr{K}_{n}$.
\end{abstract}

Keywords: Quasi-periodic solutions; coupled KdV type equations.

2010 Mathematics Subject Classification: 35C08, 47J35, 34E05

\section{Introduction}

The coupled integrable systems, which come up in many physical fields, have been studied extensively and a lot of interesting results have been given from the application field. The first coupled KdV system, which was derived by Hirota and Satsuma [1], describes interactions of two long waves with different dispersion relations. The multi-soliton solutions, conservation laws and Darboux transformation, etc., of this model were studied by many researchers [2-6]. Meanwhile, many other coupled $\mathrm{KdV}$ systems were also constructed, plenty of powerful methods were thoroughly applied to these models and lots of amazing results were obtained [7-15].

In this paper, we derive a hierarchy of coupled KdV type equations, which are composed of the positive and negative flows. The first two nontrivial members in the hierarchy are

$$
\begin{aligned}
& 0=\left(\partial^{2}-2 u \partial\right) \partial^{-1}\left(v_{t_{0}}-v_{x}\right)-2(\partial v+v \partial) \partial^{-1}\left(u_{t_{0}}-u_{x}\right), \\
& 0=4 \partial^{-1}\left(v_{t_{0}}-v_{x}\right)-(\partial+2 u) \partial^{-1}\left(u_{t_{0}}-u_{x}\right) .
\end{aligned}
$$

${ }^{*}$ Corresponding author. 


$$
\begin{aligned}
& 0=\left(\partial^{2}-2 u \partial\right) \partial^{-1}\left[2 v_{t_{1}}+v_{x x}-4(u v)_{x}\right]-2(\partial v+v \partial) \partial^{-1}\left(2 u_{t_{1}}-u_{x x}-4 u u_{x}-4 v_{x}\right), \\
& 0=4 \partial^{-1}\left[2 v_{t_{1}}+v_{x x}-4(u v)_{x}\right]-(\partial+2 u) \partial^{-1}\left(2 u_{t_{1}}-u_{x x}-4 u u_{x}-4 v_{x}\right) .
\end{aligned}
$$

The main aim of the present paper is to construct quasi-periodic solutions of the two coupled KdV type equations based on the approaches in refs. [16-20].

The outline of this paper is as follows. In section 2 we introduce the Lenard gradient and propose a hierarchy of coupled $\mathrm{KdV}$ type equations with the aid of the zero-curvature equation, in which the first two members are nonlinear evolution equations (1.1) and (1.2). In section 3 we introduce a Lax matrix from which a direct relation between the elliptic variables and the potentials is established. The two equations are separated into solvable ordinary differential equations. In section 4 we introduce the hyperelliptic Riemann surface of arithmetic genus $n$ and the Abel-Jacobi coordinates from which the corresponding flows are straightened. Finally, quasi-periodic solutions of the two coupled KdV type equations are constructed in terms of the Riemann theta function according to the asymptotic properties and the algebro-geometric characters of the meromorphic function $\phi$, the Baker-Akhiezer vector $\bar{\psi}$ and the hyperelliptic curve $\mathscr{K}_{n}$.

\section{Nonlinear Evolution Equations}

In this section, we shall derive a hierarchy of coupled KdV type equations. Let us consider spectral problem [9]

$$
\psi_{x}=U \psi, \quad \psi=\left(\begin{array}{l}
\psi_{1} \\
\psi_{2}
\end{array}\right), U=\left(\begin{array}{cc}
-\lambda+u & 2 v \\
2 & \lambda-u
\end{array}\right)
$$

where $u$ and $v$ are two potentials, $\lambda$ a constant spectral parameter. To derive the coupled KdV type hierarchy, we introduce the Lenard gradient $\left\{A_{j}, B_{j}, C_{j}\right\}$ by

$$
\begin{aligned}
& A_{-1}=-1, \quad B_{-1}=0, \quad C_{-1}=0, \\
& 2 B_{j+1}=2 u B_{j}-B_{j, x}+4 v A_{j}, \\
& 2 C_{j+1}=C_{j, x}+2 u C_{j}-4 A_{j}, \\
& A_{j, x}+2 B_{j}-2 v C_{j}=0, \quad j \geq-1 .
\end{aligned}
$$

Then $A_{j}, B_{j}, C_{j}$ are uniquely determined by recursion relation (2.3). It is easy to calculate that

$$
\begin{aligned}
& A_{0}=0, \quad B_{0}=2 v, \quad C_{0}=2, \\
& A_{1}=2 v, \quad B_{1}=2 u v-v_{x}, \quad C_{1}=2 u, \\
& A_{2}=-v_{x}+4 u v, \quad B_{2}=\frac{1}{2} v_{x x}-2 u v_{x}-u_{x} v+2 u^{2} v-4 v^{2}, \\
& C_{2}=u_{x}+2 u^{2}-4 v,
\end{aligned}
$$

Denoting $S_{j}=\left(A_{j}, B_{j}, C_{j}\right)^{\mathrm{T}}$, from the context we see that the Lenard gradient $\left\{S_{j}\right\}$ satisfies

$$
K S_{j}=J S_{j+1}, J S_{-1}=0, \quad j \geq-1
$$

in which $K$ and $J$ are two operators defined by

$$
K=\left(\begin{array}{ccc}
-4 v-\partial+2 u & 0 \\
-4 & 0 & \partial+2 u \\
\partial & 2 & -2 v
\end{array}\right), \quad J=\left(\begin{array}{ccc}
0 & 2 & 0 \\
0 & 0 & 2 \\
\partial & 2 & -2 v
\end{array}\right)
$$


Assume that the time evolution of the eigenfunction $\psi$ obeys the differential equation

$$
\psi_{t_{m}}=V^{(m)} \psi, \quad V^{(m)}=\left(\begin{array}{cc}
A^{(m)} & B^{(m)} \\
C^{(m)}-A^{(m)}
\end{array}\right)
$$

where $A^{(m)}, B^{(m)}, C^{(m)}$ are polynomials of the spectral parameter $\lambda$ with

$$
\begin{aligned}
A^{(m)} & =\widetilde{A} \lambda^{-1}+a_{m}+\sum_{j=-1}^{m-1} A_{j} \lambda^{m-j}, \\
B^{(m)} & =\widetilde{B} \lambda^{-1}+\sum_{j=-1}^{m} B_{j} \lambda^{m-j}, \\
C^{(m)} & =\widetilde{C} \lambda^{-1}+\sum_{j=-1}^{m} C_{j} \lambda^{m-j}
\end{aligned}
$$

in which $\widetilde{A}, \widetilde{B}$ and $\widetilde{C}$ are to be determined functions, and $a_{m}$ satisfies

$$
a_{m}=\frac{1}{4} C_{m, x}+\frac{1}{2} u C_{m}-\frac{1}{2} \widetilde{C} .
$$

Then the compatibility condition of (2.1) and (2.7) yields the zero-curvature equation, $U_{t_{m}}-V_{x}^{(m)}+$ $\left[U, V^{(m)}\right]=0$, which is equivalent to

$$
\begin{aligned}
& \widetilde{A}_{x}+2 \widetilde{B}-2 v \widetilde{C}=0, \\
& \widetilde{B}_{x}+4 v \widetilde{A}-2 u \widetilde{B}=0, \\
& \widetilde{C}_{x}-4 \widetilde{A}+2 u \widetilde{C}=0, \\
u_{t_{m}}= & \frac{1}{2} \partial_{x}\left(C_{m+1}-\widetilde{C}\right), \\
v_{t_{m}}= & \frac{1}{2} \partial_{x}\left(A_{m+1}-\widetilde{A}\right), \quad m \geq 0
\end{aligned}
$$

with the aid of (2.3). From (2.9), we have

$$
\begin{aligned}
& 0=\left(-\frac{1}{2} \partial^{2}+u \partial+4 v\right) \widetilde{A}+(\partial v-2 u v) \widetilde{C}, \\
& 0=-4 \widetilde{A}+(\partial+2 u) \widetilde{C}
\end{aligned}
$$

then combining (2.10) and (2.11), we obtain a new hierarchy of coupled KdV type equations

$$
\begin{aligned}
& 0=\left(\partial^{2}-2 u \partial\right) \partial^{-1}\left(2 v_{t_{m}}-A_{m+1, x}\right)-2(\partial v+v \partial) \partial^{-1}\left(2 u_{t_{m}}-C_{m+1, x}\right), \\
& 0=4 \partial^{-1}\left(2 v_{t_{m}}-A_{m+1, x}\right)-(\partial+2 u) \partial^{-1}\left(2 u_{t_{m}}-C_{m+1, x}\right) .
\end{aligned}
$$

The first two members of this hierarchy are (1.1) and (1.2) when $m=0,1$, respectively.

\section{Evolution of Elliptic Variables}

In what follows, we shall establish a relation between the elliptic variables and the potentials. Let $\chi=\left(\chi_{1}, \chi_{2}\right)^{\mathrm{T}}$ and $\varphi=\left(\varphi_{1}, \varphi_{2}\right)^{\mathrm{T}}$ be two basic solutions of (2.1) and (2.7). We introduce a Lax 
matrix

$$
W=\frac{1}{2}\left(\chi \varphi^{\mathrm{T}}+\varphi \chi^{\mathrm{T}}\right)\left(\begin{array}{cc}
0 & -1 \\
1 & 0
\end{array}\right)=\left(\begin{array}{cc}
G & F \\
H & -G
\end{array}\right)
$$

which satisfies the Lax equations

$$
W_{x}=[U, W], \quad W_{t_{m}}=\left[V^{(m)}, W\right]
$$

Therefore, $\operatorname{det} W$ is a constant independent of $x$ and $t_{m}$. Eq. (3.2) can be written as

$$
\begin{aligned}
& G_{x}=-2 F+2 v H, \\
& F_{x}=-2 \lambda F+2 u F-4 v G, \\
& H_{x}=2 \lambda H-2 u H+4 G,
\end{aligned}
$$

and

$$
\begin{aligned}
& G_{t_{m}}=-C^{(m)} F+B^{(m)} H, \\
& F_{t_{m}}=2 A^{(m)} F-2 B^{(m)} G, \\
& H_{t_{m}}=2 C^{(m)} G-2 A^{(m)} H .
\end{aligned}
$$

Suppose functions $F, G$ and $H$ are finite-order polynomials in $\lambda$ :

$$
F=\sum_{j=0}^{n+1} f_{j} \lambda^{n+1-j}, \quad G=\sum_{j=0}^{n+1} g_{j} \lambda^{n+1-j}, \quad H=\sum_{j=0}^{n+1} h_{j} \lambda^{n+1-j}
$$

Substituting (3.5) into (3.3) yields

$$
\begin{aligned}
& g_{j, x}=-2 f_{j}+2 v h_{j}, \quad f_{j, x}=-4 v g_{j}+2 u f_{j}-2 f_{j+1}, \quad h_{j, x}=4 g_{j}-2 u h_{j}+2 h_{j+1}, \\
& g_{n+1, x}=-2 f_{n+1}+2 v h_{n+1}, \quad f_{n+1, x}=-4 v g_{n+1}+2 u f_{n+1}, \quad h_{n+1, x}=4 g_{n+1}-2 u h_{n+1}, \\
& f_{0}=0, \quad h_{0}=0, \quad g_{0, x}=0,
\end{aligned}
$$

which is equivalent to

$$
\begin{aligned}
& K Q_{j}=J Q_{j+1}, \quad J Q_{0}=0, \\
& K Q_{n+1}=0,
\end{aligned}
$$

where $Q_{j}=\left(g_{j}, f_{j}, h_{j}\right)^{\mathrm{T}}, 0 \leq j \leq n+1$. It is easy to see that the equation $J Q_{0}=0$ has the general solution:

$$
Q_{0}=\alpha_{0} S_{-1}=\alpha_{0}(-1,0,0)^{\mathrm{T}}
$$

Without loss of generality, let $\alpha_{0}=1$. If we take (3.8) as a starting point, then $Q_{j}$ can be recursively determined by the relation (3.6). Acting with the operator $\left(J^{-1} K\right)^{k+1}$ upon (3.8), we obtain from 
(3.6) that

$$
Q_{k}=\sum_{j=0}^{k} \alpha_{j} S_{k-j-1}, \quad 0 \leq k \leq n+1,
$$

where $\alpha_{1}, \cdots, \alpha_{k}$ are constants of integration. The first few members in (3.9) are

$$
\begin{gathered}
Q_{1}=\left(\begin{array}{c}
\alpha_{1} \\
2 v \\
2
\end{array}\right), \quad Q_{2}=\left(\begin{array}{c}
2 v+\alpha_{2} \\
-v_{x}+2 u v-2 \alpha_{1} v \\
2 u-2 \alpha_{1}
\end{array}\right), \\
-v_{x}+4 u v-2 \alpha_{1} v+\alpha_{3} \\
Q_{3}=\left(\begin{array}{c}
\frac{1}{2} v_{x x}-v u_{x}-2 u v_{x}+2 u^{2} v-4 v^{2}+\alpha_{1}\left(v_{x}-u v\right)+2 \alpha_{2} v \\
u_{x}+2 u^{2}-4 v-2 \alpha_{1} u-2 \alpha_{2}
\end{array}\right) .
\end{gathered}
$$

If we write $F$ and $H$ as finite products which take the form:

$$
F=2 v \prod_{j=1}^{n}\left(\lambda-\mu_{j}\right), \quad H=2 \prod_{j=1}^{n}\left(\lambda-v_{j}\right),
$$

where the roots $\left\{\mu_{j}\right\}$ and $\left\{v_{j}\right\}$ are called elliptic variables. Comparing the coefficients of $\lambda^{n-1}$ in the expressions of $F$ and $H$ in (3.5) and (3.11), respectively, we arrive at

$$
\begin{gathered}
-\frac{1}{2} \frac{v_{x}}{v}+u-\alpha_{1}=-\sum_{j=1}^{n} \mu_{j}, \\
u-\alpha_{1}=-\sum_{j=1}^{n} v_{j} .
\end{gathered}
$$

Combining (3.12) and (3.13) yields

$$
\begin{gathered}
\frac{1}{2}(\ln v)_{x}=\sum_{j=1}^{n}\left(\mu_{j}-v_{j}\right), \\
u=\alpha_{1}-\sum_{j=1}^{n} v_{j}
\end{gathered}
$$

Similarly, comparing the coefficients of $\lambda^{0}$ in (3.5) and (3.11), one finds

$$
f_{n+1}=(-1)^{n} 2 v \prod_{j=1}^{n} \mu_{j}, \quad h_{n+1}=(-1)^{n} 2 \prod_{j=1}^{n} v_{j} .
$$

Since $\operatorname{det} W$ is a $(2 n+2)$ th-order polynomial in $\lambda$, whose coefficients are constants of the $x$-flow and $t_{m}$-flow, we have

$$
-\operatorname{det} W=G^{2}+F H=\prod_{j=1}^{2 n+2}\left(\lambda-\lambda_{j}\right)=R(\lambda),
$$

from which we obtain

$$
\left.G\right|_{\lambda=\mu_{k}}=\sqrt{R\left(\mu_{k}\right)},\left.\quad G\right|_{\lambda=v_{k}}=-\sqrt{R\left(v_{k}\right)} .
$$


Noticing (3.3) and (3.11), we get

$$
\begin{aligned}
& \left.F_{x}\right|_{\lambda=\mu_{k}}=-2 v \mu_{k, x} \prod_{\substack{j=1 \\
j \neq k}}^{n}\left(\mu_{k}-\mu_{j}\right)=-\left.4 v G\right|_{\lambda=\mu_{k}}, \\
& \left.H_{x}\right|_{\lambda=v_{k}}=-2 v_{k, x} \prod_{\substack{j=1 \\
j \neq k}}^{n}\left(v_{k}-v_{j}\right)=\left.4 G\right|_{\lambda=v_{k}},
\end{aligned}
$$

which means

$$
\mu_{k, x}=\frac{2 \sqrt{R\left(\mu_{k}\right)}}{\prod_{\substack{j=1 \\ j \neq k}}^{n}\left(\mu_{k}-\mu_{j}\right)}, \quad v_{k, x}=\frac{2 \sqrt{R\left(v_{k}\right)}}{\prod_{\substack{j=1 \\ j \neq k}}^{n}\left(v_{k}-v_{j}\right)}, \quad 1 \leq k \leq n
$$

In a way similar to the calculation of (3.20), we arrive at the evolution of $\left\{\mu_{j}\right\}$ and $\left\{v_{j}\right\}$ along the $t_{m}$-flow:

$$
\begin{gathered}
\mu_{k, t_{m}}=\frac{\left.B^{(m)} \sqrt{R\left(\mu_{k}\right)}\right|_{\lambda=\mu_{k}}}{v \prod_{\substack{j=1 \\
j \neq k}}^{n}\left(\mu_{k}-\mu_{j}\right)}, \\
v_{k, t_{m}}=\frac{\left.C^{(m)} \sqrt{R\left(v_{k}\right)}\right|_{\lambda=v_{k}}}{\prod_{\substack{j=1 \\
j \neq k}}^{n}\left(v_{k}-v_{j}\right)}
\end{gathered}
$$

in which $1 \leq k \leq n, 0 \leq m \leq 1$, and

$$
\begin{aligned}
& B^{(0)}=\widetilde{B} \mu_{k}^{-1}+2 v, \quad C^{(0)}=\widetilde{C} v_{k}^{-1}+2, \\
& B^{(1)}=\widetilde{B} \mu_{k}^{-1}+2 u v-v_{x}+2 v \mu_{k}, \quad C^{(1)}=\widetilde{C} v_{k}^{-1}+2 u+2 v_{k}
\end{aligned}
$$

\section{Quasi-periodic Solutions}

In the section, we shall construct quasi-periodic solutions of two coupled KdV type Eqs. (1.1) and (1.2). Noticing (3.17), one is naturally led to introduce the hyperelliptic curve $\mathscr{K}_{n}$ of arithmetic genus $n$ defined by

$$
\mathscr{K}_{n}: \quad y^{2}-R(\lambda)=0 .
$$

The curve $\mathscr{K}_{n}$ can be compactified by joining two points at infinity, $P_{\infty \pm}$, where $P_{\infty+} \neq P_{\infty-}$. For notational simplicity the compactification is also denoted by $\mathscr{K}_{n}$. Here we assume that the zeros $\lambda_{j}$ of $R(\lambda)$ in (3.17) are mutually distinct, which means $\lambda_{j} \neq \lambda_{k}$, for $j \neq k, 1 \leq j, k \leq 2 n+2$. Then the hyperelliptic curve $\mathscr{K}_{n}$ becomes nonsingular. According to the definition of $\mathscr{K}_{n}$, we can lift the 
roots $\left\{\mu_{j}\right\}$ and $\left\{v_{j}\right\}$ to $\mathscr{K}_{n}$ by introducing

$$
\begin{aligned}
& \hat{\mu}_{j}\left(x, t_{m}\right)=\left(\mu_{j}\left(x, t_{m}\right), G\left(\mu_{j}\left(x, t_{m}\right), x, t_{m}\right)\right), \\
& \hat{v}_{j}\left(x, t_{m}\right)=\left(v_{j}\left(x, t_{m}\right),-G\left(v_{j}\left(x, t_{m}\right), x, t_{m}\right)\right),
\end{aligned}
$$

where $j=1, \ldots, n,\left(x, t_{m}\right) \in \mathbb{R}^{2}$. Moreover, from (4.1) we know that

$$
y^{2}=G^{2}+F H
$$

that is

$$
(y+G)(y-G)=F H,
$$

then we can define the meromorphic function $\phi\left(\cdot, x, t_{m}\right)$ on $\mathscr{K}_{n}$ :

$$
\phi\left(P, x, t_{m}\right)=\frac{y+G}{F}=\frac{H}{y-G},
$$

where $P=(\lambda, y) \in \mathscr{K}_{n} \backslash\left\{P_{\infty+}, P_{\infty-}\right\}$. Hence the divisor of $\phi\left(\cdot, x, t_{m}\right)$ reads [17,21]

$$
\left(\phi\left(\cdot, x, t_{m}\right)\right)=D_{\hat{v}\left(x, t_{m}\right)}-D_{\hat{\mu}\left(x, t_{m}\right)}+P_{\infty-}-P_{\infty+},
$$

with $D_{\hat{\mu}\left(x, t_{m}\right)}=\sum_{j=1}^{n} \hat{\mu}_{j}\left(x, t_{m}\right), D_{\hat{v}\left(x, t_{m}\right)}=\sum_{j=1}^{n} \hat{v}_{j}\left(x, t_{m}\right)$.

Based on the definition of meromorphic function $\phi\left(\cdot, x, t_{m}\right)$ in (4.4), the spectral problem (2.1) and the auxiliary problem (2.7), we can define the Baker-Akhiezer vector $\bar{\psi}\left(\cdot, x, x_{0}, t_{m}, t_{m, 0}\right)$ on $\mathscr{K}_{n} \backslash\left\{P_{\infty+}, P_{\infty-}\right\}$ by

$$
\bar{\psi}\left(P, x, x_{0}, t_{m}, t_{m, 0}\right)=\left(\begin{array}{l}
\psi_{1}\left(P, x, x_{0}, t_{m}, t_{m, 0}\right) \\
\psi_{2}\left(P, x, x_{0}, t_{m}, t_{m, 0}\right)
\end{array}\right)
$$

where

$$
\begin{aligned}
& \psi_{1}\left(P, x, x_{0}, t_{m}, t_{m, 0}\right)= \exp \left(\int_{x_{0}}^{x}\left(-\lambda+u\left(x^{\prime}, t_{m}\right)-2 v\left(x^{\prime}, t_{m}\right) \phi\left(P, x^{\prime}, t_{m}\right)\right) d x^{\prime}\right. \\
&\left.+\int_{t_{m, 0}}^{t_{m}}\left(A^{(m)}\left(\lambda, x_{0}, s\right)-B^{(m)}\left(\lambda, x_{0}, s\right) \phi\left(P, x_{0}, s\right)\right) d s\right), \\
& \psi_{2}\left(P, x, x_{0}, t_{m}, t_{m, 0}\right)=-\phi\left(P, x, t_{m}\right) \psi_{1}\left(P, x, x_{0}, t_{m}, t_{m, 0}\right)
\end{aligned}
$$

with $P \in \mathscr{K}_{n} \backslash\left\{P_{\infty+}, P_{\infty-}\right\},\left(x, t_{m}\right),\left(x_{0}, t_{m, 0}\right) \in \mathbb{R}^{2}$.

Next, let us introduce the Riemann surface $\Gamma$ of the hyperelliptic curve $\mathscr{K}_{n}$ and equip $\Gamma$ with canonical basis cycles: $a_{1}, \cdots, a_{n} ; b_{1}, \cdots, b_{n}$, which are independent and have intersection numbers 
as follows

$$
a_{i} \circ a_{j}=0, \quad b_{i} \circ b_{j}=0, \quad a_{i} \circ b_{j}=\delta_{i j} .
$$

For the present, we will choose our basis as the following set

$$
\tilde{\omega}_{l}=\frac{\lambda^{l-1} d \lambda}{\sqrt{R(\lambda)}}, \quad 1 \leq l \leq n
$$

which are $n$ linearly independent homomorphic differentials on $\Gamma$. By using the cycles $a$ and $b$, the period matrices $A$ and $B$ can be constructed from

$$
A_{i j}=\int_{a_{j}} \tilde{\omega}_{i}, \quad B_{i j}=\int_{b_{j}} \tilde{\omega}_{i}
$$

It is possible to show that matrices $A$ and $B$ are invertible [21,22]. Now we define the matrices $C$ and $\tau$ by $C=A^{-1}, \tau=A^{-1} B$. The matrix $\tau$ can be shown to be symmetric $\left(\tau_{i j}=\tau_{j i}\right)$ and it has positive definite imaginary part $(\operatorname{Im} \tau>0)$. If we normalize $\tilde{\omega}_{l}$ into the new basis $\omega_{j}$,

$$
\omega_{j}=\sum_{l=1}^{n} C_{j l} \tilde{\omega}_{l}, \quad 1 \leq j \leq n
$$

then we have

$$
\int_{a_{i}} \omega_{j}=\sum_{l=1}^{n} C_{j l} \int_{a_{i}} \tilde{\omega}_{l}=\delta_{j i}, \quad \int_{b_{i}} \omega_{j}=\tau_{j i}
$$

Define the Abel-Jacobi coordinates

$$
\begin{aligned}
& \rho_{j}^{(1)}\left(x, t_{m}\right)=\sum_{k=1}^{n} \int_{P_{0}}^{\hat{\mu}_{k}\left(x, t_{m}\right)} \omega_{j}=\sum_{k=1}^{n} \sum_{l=1}^{n} C_{j l} \int_{\lambda\left(P_{0}\right)}^{\mu_{k}} \frac{\lambda^{l-1} d \lambda}{\sqrt{R(\lambda)}}, \\
& \rho_{j}^{(2)}\left(x, t_{m}\right)=\sum_{k=1}^{n} \int_{P_{0}}^{\hat{v}_{k}\left(x, t_{m}\right)} \omega_{j}=\sum_{k=1}^{n} \sum_{l=1}^{n} C_{j l} \int_{\lambda\left(P_{0}\right)}^{v_{k}} \frac{\lambda^{l-1} d \lambda}{\sqrt{R(\lambda)}},
\end{aligned}
$$

where $1 \leq j \leq n$. Without loss of generality, we choose the branch point $P_{0}=\left(\lambda_{j_{0}}, 0\right), j_{0} \in$ $\{1, \cdots, 2 n+2\}$, as a convenient base point, and $\lambda\left(P_{0}\right)$ is its local coordinate. From (3.20), we obtain

$$
\begin{aligned}
& \partial_{x} \rho_{j}^{(1)}=\sum_{l=1}^{n} \sum_{k=1}^{n} C_{j l} \frac{\mu_{k}^{l-1} \mu_{k, x}}{\sqrt{R\left(\mu_{k}\right)}}=\sum_{l=1}^{n} \sum_{k=1}^{n} \frac{2 C_{j l} \mu_{k}^{l-1}}{\prod_{\substack{i=1 \\
i \neq k}}^{n}\left(\mu_{k}-\mu_{i}\right)}, \\
& \partial_{x} \rho_{j}^{(2)}=\sum_{l=1}^{n} \sum_{k=1}^{n} C_{j l} \frac{v_{k}^{l-1} v_{k, x}}{\sqrt{R\left(v_{k}\right)}}=\sum_{l=1}^{n} \sum_{k=1}^{n} \frac{2 C_{j l} v_{k}^{l-1}}{\prod_{\substack{i=1 \\
i \neq k}}^{n}\left(v_{k}-v_{i}\right)}
\end{aligned}
$$

which implies

$$
\partial_{x} \rho_{j}^{(1)}=2 C_{j n}, \quad \partial_{x} \rho_{j}^{(2)}=2 C_{j n}, \quad 1 \leq j \leq n,
$$


with the help of the following equalities

$$
\sum_{k=1}^{N} \frac{\mu_{k}^{l-1}}{\prod_{i \neq k}\left(\mu_{k}-\mu_{i}\right)}=\left\{\begin{array}{cc}
\delta_{l N}, & 1 \leq l \leq N, \\
\sum_{i_{1}+\cdots+i_{N}=l-N, i_{j} \geq 0} \mu_{1}^{i_{1}} \ldots \mu_{N}^{i_{N}}, & l>N .
\end{array}\right.
$$

In a similar way, we obtain from (3.21)-(3.23) that

$$
\begin{aligned}
& \partial_{t_{0}} \rho_{j}^{(1)}=(-1)^{n+1} 2 C_{j 1}+2 C_{j n}=Y_{j}^{(0)}, \\
& \partial_{t_{0}} \rho_{j}^{(2)}=(-1)^{n+1} 2 C_{j 1}+2 C_{j n}=Y_{j}^{(0)}, \\
& \partial_{t_{1}} \rho_{j}^{(1)}=(-1)^{n+1} 2 C_{j 1}+2 C_{j, n-1}+2 \alpha_{1} C_{j n}=Y_{j}^{(1)}, \\
& \partial_{t_{1}} \rho_{j}^{(2)}=(-1)^{n+1} 2 C_{j 1}+2 C_{j, n-1}+2 \alpha_{1} C_{j n}=Y_{j}^{(1)} .
\end{aligned}
$$

In fact, in order to straighten the time-dependent flow, we need to assume that

$$
(\widetilde{A}, \widetilde{B}, \widetilde{C})^{\mathrm{T}}=Q_{n+1}
$$

It's easy to see that our assumption is reasonable, because from (2.9) and (3.7) we know that $(\widetilde{A}, \widetilde{B}, \widetilde{C})^{\mathrm{T}} \in \operatorname{ker} K$ and $Q_{n+1} \in \operatorname{ker} K$, respectively. Then under our assumption, $0=(\widetilde{A}, \widetilde{B}, \widetilde{C})^{\mathrm{T}}-$ $Q_{n+1} \in \operatorname{ker} K$ apparently holds according to the expression of $K$. With the help of (4.16) and the following equality

$$
\sum_{k=1}^{n} \frac{\mu_{k}^{-1}}{\prod_{\substack{i=1 \\ i \neq k}}^{n}\left(\mu_{k}-\mu_{i}\right)}=\frac{1}{\prod_{i=1}^{n} \mu_{i}}
$$

we can obtain (4.17)-(4.20).

Let $\mathscr{T}$ be the lattice generated by $2 n$ vectors $\delta_{j}, \tau_{j}$, where $\delta_{j}=(\underbrace{0, \ldots, 0}_{j-1}, 1, \underbrace{0, \ldots, 0}_{n-j})$ and $\tau_{j}=\tau \delta_{j}$.

The complex torus $\mathscr{J}=\mathbb{C}^{n} / \mathscr{T}$ is called Jacobian variety of $\Gamma$. Now we introduce the Abel map $\mathscr{A}(P): \operatorname{Div}(\Gamma) \rightarrow \mathscr{J}$

$$
\mathscr{A}(P)=\int_{P_{0}}^{P} \underline{\omega}, \quad \mathscr{A}\left(\sum n_{k} P_{k}\right)=\sum n_{k} \mathscr{A}\left(P_{k}\right)
$$

where $P, P_{k} \in \mathscr{K}_{n}, \underline{\omega}=\left(\omega_{1}, \ldots, \omega_{n}\right)$. Consider two special divisors $\sum_{k=1}^{n} P_{k}^{(i)}, i=1,2$, and

$$
\mathscr{A}\left(\sum_{k=1}^{n} P_{k}^{(i)}\right)=\sum_{k=1}^{n} \mathscr{A}\left(P_{k}^{(i)}\right)=\sum_{k=1}^{n} \int_{P_{0}}^{P_{k}^{(i)}} \underline{\omega}=\underline{\rho}^{(i)},
$$

with $P_{k}^{(1)}=\hat{\mu}_{k}\left(x, t_{m}\right)$ and $P_{k}^{(2)}=\hat{v}_{k}\left(x, t_{m}\right)$, whose components are

$$
\sum_{k=1}^{n} \int_{P_{0}}^{P_{k}^{(i)}} \omega_{j}=\rho_{j}^{(i)}, \quad 1 \leq j \leq n, \quad i=1,2 .
$$


The Riemann theta function is defined as $[17,21,22]$

$$
\theta(P, D)=\theta(\underline{\Lambda}-\mathscr{A}(P)+\mathscr{A}(D)),
$$

where $P \in \mathscr{K}_{n}, D \in \operatorname{Div}(\Gamma)$, and $\underline{\Lambda}=\left(\Lambda_{1}, \ldots, \Lambda_{n}\right)$ is defined by

$$
\Lambda_{j}=\frac{1}{2}\left(1+\tau_{j j}\right)-\sum_{\substack{i=1 \\ i \neq j}}^{n} \int_{a_{i}} \omega_{i} \int_{P_{0}}^{P} \omega_{j}, \quad j=1, \ldots, n .
$$

Then according to (4.22) and the definition of Riemann theta function in (4.23), we have

$$
\begin{aligned}
& \theta\left(P, D_{\hat{\mu}\left(x, t_{m}\right)}\right)=\theta\left(\underline{\Lambda}-\mathscr{A}(P)+\underline{\rho}^{(1)}\right), \\
& \theta\left(P, D_{\hat{v}\left(x, t_{m}\right)}\right)=\theta\left(\underline{\Lambda}-\mathscr{A}(P)+\underline{\rho}^{(2)}\right) .
\end{aligned}
$$

Based on (4.15) and (4.17)-(4.20), we have the following lemma.

Lemma 1. (Straightening out of the flow)

$$
\begin{aligned}
& \partial_{x} \underline{\rho}^{(1)}=2 \underline{C}_{n}, \quad \partial_{x} \underline{\rho}^{(2)}=2 \underline{C}_{n}, \\
& \partial_{t_{m}} \underline{\rho}^{(1)}=\underline{Y}^{(m)}, \partial_{t_{m}} \underline{\rho}^{(2)}=\underline{Y}^{(m)}, \quad 0 \leq m \leq 1,
\end{aligned}
$$

where $\underline{\rho}^{(i)}=\left(\rho_{1}^{(i)}, \ldots, \rho_{n}^{(i)}\right), i=1,2, \underline{C}_{n}=\left(C_{1 n}, \ldots, C_{n n}\right), \underline{Y}^{(m)}=\left(Y_{1}^{(m)}, \ldots, Y_{n}^{(m)}\right)$.

In order to derive the quasi-periodic solutions of coupled KdV type Eqs. (1.1) and (1.2), now we turn to the asymptotic properties of the meromorphic function $\phi$.

Lemma 2. Suppose that $u\left(x, t_{m}\right), v\left(x, t_{m}\right) \in C^{\infty}\left(\mathbb{R}^{2}\right)$ satisfy the hierarchy of coupled $K d V$ type equations (2.12). Let $\lambda_{j} \in \mathbb{C} \backslash\{0\},(1 \leq j \leq 2 n+2)$, and $P=(\lambda, y) \in \mathscr{K}_{n} \backslash\left\{P_{\infty+}, P_{\infty_{-}}\right\}$. Then

$$
\phi \underset{\zeta \rightarrow 0}{=} \begin{cases}-\frac{1}{v} \zeta^{-1}+\left(\frac{u}{v}-\frac{v_{x}}{2 v^{2}}\right)+O(\zeta) & \text { as } P \rightarrow P_{\infty+}, \\ \zeta+O\left(\zeta^{2}\right) & \text { as } P \rightarrow P_{\infty-} .\end{cases}
$$

Proof. First, from (3.17) we have

$$
\begin{aligned}
G^{2}+F H & =\prod_{j=1}^{2 n+2}\left(\lambda-\lambda_{j}\right) \\
& =\lambda^{2 n+2}-\sum_{j=1}^{2 n+2} \lambda_{j} \lambda^{2 n+1}+\sum_{j<k} \lambda_{j} \lambda_{k} \lambda^{2 n}-\sum_{i<j<k} \lambda_{i} \lambda_{j} \lambda_{k} \lambda^{2 n-1}+O\left(\lambda^{2 n-2}\right) .
\end{aligned}
$$

On the other hand, according to (3.5) we obtain

$$
\begin{aligned}
G^{2}+F H= & g_{0}^{2} \lambda^{2 n+2}+2 g_{0} g_{1} \lambda^{2 n+1}+\left(g_{1}^{2}+2 g_{0} g_{2}+f_{1} h_{1}\right) \lambda^{2 n} \\
& +\left(2 g_{0} g_{3}+2 g_{1} g_{2}+f_{1} h_{2}+f_{2} h_{1}\right) \lambda^{2 n-1}+O\left(\lambda^{2 n-2}\right) .
\end{aligned}
$$

Then, comparing the coefficients of $\lambda$ with the same power in (4.27) and (4.28), and combining (3.10), (3.14) and (3.15), one computes

$$
\alpha_{1}=-\frac{1}{2} \sum_{j=1}^{2 n+2} \lambda_{j}, \quad \alpha_{2}=\frac{1}{2}\left(\sum_{j<k} \lambda_{j} \lambda_{k}-\frac{1}{4}\left(\sum_{j=1}^{2 n+2} \lambda_{j}\right)^{2}\right) .
$$


Introducing the local coordinate $\zeta=\lambda^{-1}$ near $P_{\infty \pm}$, from (3.17) and (4.1), we have

$$
y=\mp \prod_{j=1}^{2 n+2}\left(\lambda-\lambda_{j}\right)^{\frac{1}{2}}=\mp \zeta^{-n-1} \prod_{j=1}^{2 n+2}\left(1-\lambda_{j} \zeta\right)^{\frac{1}{2}} \quad \text { as } P \rightarrow P_{\infty \pm} .
$$

Since

$$
\begin{aligned}
& \prod_{j=1}^{2 n+2}\left(1-\lambda_{j} \zeta\right)^{\frac{1}{2}} \underset{\zeta \rightarrow 0}{=} \prod_{j=1}^{2 n+2}\left(1-\frac{1}{2} \lambda_{j} \zeta-\frac{1}{8} \lambda_{j}^{2} \zeta^{2}-\frac{1}{16} \lambda_{j}^{3} \zeta^{3}+O\left(\zeta^{4}\right)\right) \\
& \underset{\zeta \rightarrow 0}{=} 1+\varepsilon_{1} \zeta+\varepsilon_{2} \zeta^{2}+O\left(\zeta^{3}\right) \quad \text { as } P \rightarrow P_{\infty \pm},
\end{aligned}
$$

where

$$
\varepsilon_{1}=-\frac{1}{2} \sum_{j=1}^{2 n+2} \lambda_{j}, \quad \varepsilon_{2}=\frac{1}{2}\left(\sum_{j<k} \lambda_{j} \lambda_{k}-\frac{1}{4}\left(\sum_{j=1}^{2 n+2} \lambda_{j}\right)^{2}\right)
$$

Therefore,

$$
y \underset{\zeta \rightarrow 0}{=} \mp \zeta^{-n-1}\left(1+\varepsilon_{1} \zeta+\varepsilon_{2} \zeta^{2}+O\left(\zeta^{3}\right)\right) \quad \text { as } P \rightarrow P_{\infty \pm} .
$$

From (3.11) and (3.5), we can derive

$$
\begin{aligned}
& F^{-1}=\frac{1}{2 v} \prod_{j=1}^{n}\left(\lambda-\mu_{j}\right)^{-1} \underset{\zeta \rightarrow 0}{=} \frac{1}{2 v} \zeta^{n} \prod_{j=1}^{n}\left(1-\mu_{j} \zeta\right)^{-1} \\
& \underset{\zeta \rightarrow 0}{=} \frac{1}{2 v} \zeta^{n}\left(1+\sum_{j=1}^{n} \mu_{j} \zeta+O\left(\zeta^{2}\right)\right) \quad \text { as } P \rightarrow P_{\infty \pm} \text {, } \\
& G=g_{0} \lambda^{n+1}+g_{1} \lambda^{n}+g_{2} \lambda^{n-1}+O\left(\lambda^{n-2}\right) \\
& \underset{\zeta \rightarrow 0}{=} \zeta^{-n-1}\left(g_{0}+g_{1} \zeta+g_{2} \zeta^{2}+O\left(\zeta^{3}\right)\right) \quad \text { as } P \rightarrow P_{\infty \pm} .
\end{aligned}
$$

Then according to the definition of $\phi$ in (4.4), we finally obtain that

$$
\begin{aligned}
& \phi= \frac{y+G}{F} \\
& \underset{\zeta \rightarrow 0}{=} \frac{1}{2 v} \zeta^{-1}\left(\mp\left(1+\varepsilon_{1} \zeta+\varepsilon_{2} \zeta^{2}+O\left(\zeta^{3}\right)\right)+g_{0}+g_{1} \zeta+g_{2} \zeta^{2}+O\left(\zeta^{3}\right)\right) \\
& \times\left(1+\sum_{j=1}^{n} \mu_{j} \zeta+O\left(\zeta^{2}\right)\right) \\
& \underset{\zeta \rightarrow 0}{=}\left\{\begin{array}{lc}
-\frac{1}{v} \zeta^{-1}+\left(\frac{u}{v}-\frac{v_{x}}{2 v^{2}}\right)+O(\zeta) & \text { as } P \rightarrow P_{\infty+}, \\
\zeta+O\left(\zeta^{2}\right) & \text { as } P \rightarrow P_{\infty-},
\end{array}\right.
\end{aligned}
$$

which proves this lemma. 
Lemma 3. Under the same supposition in Lemma 2, in the special case of (3.9) when $\alpha_{0}=$ $1, \alpha_{k}=0,1 \leq k \leq n+1$, we have that

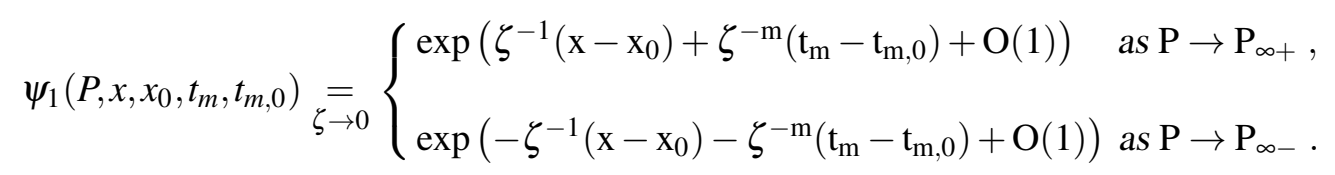

Proof. According to (3.5), in the special case of (3.9) when $\alpha_{0}=1, \alpha_{k}=0,1 \leq k \leq n+1$, we derive that $f_{j}=B_{j-1}, 0 \leq j \leq n+1$. Introducing the local coordinate $\zeta=\lambda^{-1}$ near $P_{\infty \pm}$, we obtain from (4.26) that

$$
\begin{aligned}
& \exp \left(\int_{x_{0}}^{x}(-\lambda+u-2 v \phi) d x^{\prime}\right)=\exp \left(\int_{x_{0}}^{x}\left(-\zeta^{-1}+u-2 v \phi\right) d x^{\prime}\right) \\
& \underset{\zeta \rightarrow 0}{=} \begin{cases}\exp \left[\int_{x_{0}}^{x}\left(-\zeta^{-1}+u-2 v\left(-\frac{1}{v} \zeta^{-1}+\frac{u}{v}-\frac{v_{x}}{2 v^{2}}+O(\zeta)\right)\right) d x^{\prime}\right] & \text { as } P \rightarrow P_{\infty+}, \\
\left.\exp \left[\int_{x_{0}}^{x}\left(-\zeta^{-1}+u-2 v \zeta+O\left(\zeta^{2}\right)\right)\right) d x^{\prime}\right] & \text { as } P \rightarrow P_{\infty-},\end{cases} \\
& \underset{\zeta \rightarrow 0}{=} \begin{cases}\exp \left(\zeta^{-1}\left(x-x_{0}\right)+O(1)\right) & \text { as } P \rightarrow P_{\infty+}, \\
\left(\exp \left(\int_{x_{0}}^{x} u\left(x^{\prime}, t_{m}\right) d x^{\prime}\right)+O(\zeta)\right) \exp \left(-\zeta^{-1}\left(x-x_{0}\right)+O(\zeta)\right) & \text { as } P \rightarrow P_{\infty-},\end{cases}
\end{aligned}
$$


Then, in the special case of (3.9), combining (2.10), (3.4), (4.4) and (4.30) yields

$$
\begin{aligned}
& \exp \left(\int_{t_{m, 0}}^{t_{m}}\left(A^{(m)}-B^{(m)} \phi\right) d s\right)=\exp \left(\int_{t_{m, 0}}^{t_{m}}\left(-\frac{y}{F} B^{(m)}+\frac{A^{(m)} F-B^{(m)} G}{F}\right) d s\right) \\
& =\exp \left(\int_{t_{m, 0}}^{t_{m}}\left(-\frac{y}{F} B^{(m)}+\frac{1}{2} \frac{F_{t_{m}}}{F}\right) d s\right) \\
& \underset{\zeta \rightarrow 0}{=\exp }\left[\int_{t_{m, 0}}^{t_{m}}\left( \pm \zeta^{-n-1}(1+O(\zeta)) \times \frac{\widetilde{B} \zeta+\sum_{j=-1}^{m} B_{j} \zeta^{j-m}}{\sum_{j=0}^{n+1} f_{j} \zeta^{j-n-1}}+\frac{v_{t_{m}}}{2 v}+O(\zeta)\right) d s\right] \\
& \underset{\zeta \rightarrow 0}{=\exp }\left[\int_{t_{m, 0}}^{t_{m}}\left( \pm \zeta^{-m}(1+O(\zeta)) \times \frac{\widetilde{B} \zeta^{m+1}+\sum_{j=-1}^{m} B_{j} \zeta^{j}}{\sum_{j=0}^{n+1} f_{j} \zeta^{j}}+\frac{v_{t_{m}}}{2 v}+O(\zeta)\right] d s\right]
\end{aligned}
$$

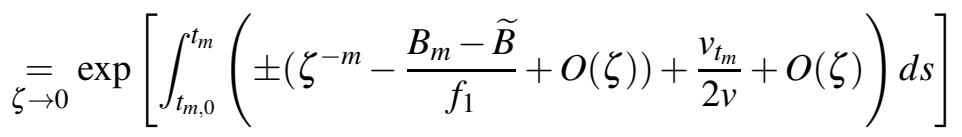

$$
\begin{aligned}
& \underset{\zeta \rightarrow 0}{=} \exp \left[\int_{t_{m, 0}}^{t_{m}}\left( \pm \zeta^{-m}+O(1)\right) d s\right] \\
& \underset{\zeta \rightarrow 0}{=} \exp \left( \pm \zeta^{-m}\left(t_{m}-t_{m, 0}\right)+O(1)\right) \quad \text { as } P \rightarrow P_{\infty \pm} .
\end{aligned}
$$

Hence, according to the definition of $\psi_{1}$ in (4.7), (4.36) and (4.37), we can arrive at (4.35).

Next, we shall derive the representation of $\phi, \bar{\psi}, u$ and $v$ in terms of the Riemann theta function. Let $\omega_{P_{\infty-}, P_{\infty+}}^{(3)}$ be the normal differential of the third kind holomorphic on $\mathscr{K}_{n} \backslash\left\{P_{\infty+}, P_{\infty-}\right\}$ with simple poles at $P_{\infty-}$ and $P_{\infty+}$ and residues 1 and -1 , respectively, which can be expressed as

$$
\omega_{P_{\infty}-P_{\infty+}}^{(3)}=-\frac{1}{y} \prod_{j=1}^{n}\left(\lambda-\beta_{j}\right) d \lambda
$$

where $\beta_{j} \in \mathbb{C}, j=1, \ldots, n$, are constants that are determined by

$$
\int_{a_{j}} \omega_{P_{\infty-}, P_{\infty+}}^{(3)}=0, \quad j=1, \ldots, n
$$


If the local coordinate near $P_{\infty \pm}$ is given by $\zeta=\lambda^{-1}$, then we have the asymptotic expansions of $\omega_{P_{\infty}, P_{\infty+}}^{(3)}$ near $P_{\infty \pm}$ :

$$
\begin{aligned}
& \omega_{P_{\infty}, P_{\infty+}}^{(3)} \underset{\zeta \rightarrow 0}{=} \pm \zeta^{n+1} \prod_{j=1}^{2 n+2}\left(1-\lambda_{j} \zeta\right)^{-\frac{1}{2}} \cdot\left(-\zeta^{-n-2} \prod_{j=1}^{n}\left(1-\beta_{j} \zeta\right)\right) d \zeta \\
& \underset{\zeta \rightarrow 0}{=} \mp \zeta^{-1} \prod_{j=1}^{2 n+2}\left(1-\lambda_{j} \zeta\right)^{-\frac{1}{2}} \cdot \prod_{j=1}^{n}\left(1-\beta_{j} \zeta\right) d \zeta \\
& \underset{\zeta \rightarrow 0}{=} \mp\left(\zeta^{-1}+O(1)\right) d \zeta \quad \text { as } P \rightarrow P_{\infty \pm} \text {. }
\end{aligned}
$$

Therefore,

$$
\int_{P_{0}}^{P} \omega_{P_{\infty}, P_{\infty+}}^{(3)} \underset{\zeta \rightarrow 0}{=} \mp\left(\ln \zeta-\ln \omega_{0}+O(\zeta)\right) \quad \text { as } P \rightarrow P_{\infty \pm}
$$

for some constant $\omega_{0} \in \mathbb{C}$.

Next, let $\omega_{P_{\infty}, r}^{(2)}, r \in \mathbb{N}_{0}$, be normalized differentials of the second kind with a unique pole at $P_{\infty \pm}$ and principal part $P_{\infty \pm}$ near $-\zeta^{-2-r} d \zeta$, satisfying

$$
\int_{a_{j}} \omega_{P_{\infty \pm}, r}^{(2)}=0, \quad j=1,2, \ldots, n .
$$

Then we can define $\Omega_{0}^{(2)}$ and $\Omega_{m-1}^{(2)}$ by

$$
\begin{gathered}
\Omega_{0}^{(2)}=\omega_{P_{\infty+}, 0}^{(2)}-\omega_{P_{\infty-}, 0}^{(2)}, \\
\Omega_{m-1}^{(2)}=\sum_{q=0}^{m-1} \alpha_{m-1-q}(q+1)\left(\omega_{P_{\infty}, q}^{(2)}-\omega_{P_{\infty}, q}^{(2)}\right),
\end{gathered}
$$

where $\alpha_{m-1-q}$ are the integral constants in (3.9). Therefore,

$$
\begin{gathered}
\int_{a_{j}} \Omega_{0}^{(2)}=0, \quad \int_{a_{j}} \Omega_{m-1}^{(2)}=0, \quad j=1,2, \ldots, n . \\
\int_{P_{0}}^{P} \Omega_{0}^{(2)} \underset{\zeta \rightarrow 0}{=} \pm\left(\zeta^{-1}+e_{0,0}+e_{0,1} \zeta+O\left(\zeta^{2}\right)\right) \quad \text { as } P \rightarrow P_{\infty \pm}, \\
\int_{P_{0}}^{P} \Omega_{m-1}^{(2)} \underset{\zeta \rightarrow 0}{=} \pm\left(\sum_{q=0}^{m-1} \alpha_{m-1-q} \zeta^{-1-q}+e_{m-1,0}+O(\zeta)\right) \quad \text { as } P \rightarrow P_{\infty \pm}
\end{gathered}
$$

for some constants $e_{0,0}, e_{0,1}, e_{m-1,0} \in \mathbb{C}$.

If $D_{\hat{\mu}\left(x, t_{m}\right)}$ and $D_{\hat{v}\left(x, t_{m}\right)}$ in (4.5) are assumed to be nonspecial [21], then according to the Riemann's vanishing theorem [17,21], the definition and asymptotic properties of the meromorphic 
function $\phi\left(P, x, t_{m}\right), \phi$ has the expressions of the following type:

$$
\phi\left(P, x, t_{m}\right)=N\left(x, t_{m}\right) \frac{\theta\left(P, D_{\hat{v}\left(x, t_{m}\right)}\right)}{\theta\left(P, D_{\hat{\mu}\left(x, t_{m}\right)}\right)} \exp \left(\int_{P_{0}}^{P} \omega_{P_{\infty}, P_{\infty+}}^{(3)}\right),
$$

where $N\left(x, t_{m}\right)$ is independent of $P \in \mathscr{K}_{n}$.

Theorem 1. Let $P=(\lambda, y) \in \mathscr{K}_{n} \backslash\left\{P_{\infty+}, P_{\infty-}\right\},\left(x, t_{m}\right),\left(x_{0}, t_{m, 0}\right) \in M$, where $M \subseteq \mathbb{R}^{2}$ is open and connected. Suppose $u, v \in C^{\infty}(M)$ satisfy the hierarchy of coupled $K d V$ type equations (2.12), and assume that $\lambda_{j}, 1 \leq j \leq 2 n+2$, in (3.17) satisfy $\lambda_{j} \in \mathbb{C} \backslash\{0\}$ and $\lambda_{j} \neq \lambda_{k}$ as $j \neq k$. Moreover, suppose that $D_{\hat{\mu}\left(x, t_{m}\right)}$, or equivalently, $D_{\hat{v}\left(x, t_{m}\right)}$ is nonspecial for $\left(x, t_{m}\right) \in M$. Then $\phi, \psi_{1}, \psi_{2}$ admit the following representation

$$
\begin{aligned}
\phi\left(P, x, t_{m}\right)= & w_{0} \frac{\theta\left(P_{\infty-}, D_{\hat{\mu}\left(x, t_{m}\right)}\right) \theta\left(P, D_{\hat{v}\left(x, t_{m}\right)}\right)}{\theta\left(P_{\infty-}, D_{\hat{v}\left(x, t_{m}\right)}\right) \theta\left(P, D_{\hat{\mu}\left(x, t_{m}\right)}\right)} \times \exp \left(\int_{\mathrm{P}_{0}}^{\mathrm{P}} \omega_{\mathrm{P}_{\infty-}, \mathrm{P}_{\infty+}}^{(3)}\right) \\
\psi_{1}\left(P, x, t_{m}, x_{0}, t_{m, 0}\right)= & \frac{\theta\left(P_{\infty+}, D_{\hat{\mu}\left(x_{0}, t_{m, 0}\right)}\right) \theta\left(P, D_{\hat{\mu}\left(x, t_{m}\right)}\right)}{\theta\left(P_{\infty+}, D_{\hat{\mu}\left(x, t_{m}\right)}\right) \theta\left(P, D_{\hat{\mu}\left(x_{0}, t_{m, 0}\right)}\right)} \\
& \times \exp \left(\left(\int_{\mathrm{P}_{0}}^{\mathrm{P}} \Omega_{0}^{(2)}-\mathrm{e}_{0,0}\right)\left(\mathrm{x}-\mathrm{x}_{0}\right)+\left(\int_{\mathrm{P}_{0}}^{\mathrm{P}} \Omega_{\mathrm{m}-1}^{(2)}-\mathrm{e}_{\mathrm{m}-1,0}\right)\left(\mathrm{t}_{\mathrm{m}}-\mathrm{t}_{\mathrm{m}, 0}\right)\right) \\
\psi_{2}\left(P, x, t_{m}, x_{0}, t_{m, 0}\right)= & -w_{0} \frac{\theta\left(P_{\infty+}, D_{\hat{\mu}\left(x_{0}, t_{m, 0}\right)}\right) \theta\left(P_{\infty-}, D_{\hat{\mu}\left(x, t_{m}\right)}\right) \theta\left(P, D_{\hat{v}\left(x, t_{m}\right)}\right)}{\theta\left(P_{\infty+}, D_{\hat{\mu}\left(x, t_{m}\right)}\right) \theta\left(P_{\infty-}, D_{\hat{v}\left(x, t_{m}\right)}\right) \theta\left(P, D_{\hat{\mu}\left(x_{0}, t_{m, 0}\right)}\right)} \\
\times \exp \left(\int_{\mathrm{P}_{0}}^{\mathrm{P}} \omega_{\mathrm{P}_{\infty-}, \mathrm{P}_{\infty+}}^{(3)}\right. & \left.+\left(\int_{\mathrm{P}_{0}}^{\mathrm{P}} \Omega_{0}^{(2)}+\mathrm{e}_{0,0}\right)\left(\mathrm{x}-\mathrm{x}_{0}\right)+\left(\int_{\mathrm{P}_{0}}^{\mathrm{P}} \Omega_{\mathrm{m}-1}^{(2)}+\mathrm{e}_{\mathrm{m}-1,0}\right)\left(\mathrm{t}_{\mathrm{m}}-\mathrm{t}_{\mathrm{m}, 0}\right)\right)
\end{aligned}
$$

Finally, $u\left(x, t_{m}\right), v\left(x, t_{m}\right)$ are of the form

$$
\begin{gathered}
u\left(x, t_{m}\right)=\partial_{x} \ln \frac{\theta\left(P_{\infty+}, D_{\hat{\mu}\left(x_{0}, t_{m, 0}\right)}\right) \theta\left(P_{\infty-}, D_{\hat{\mu}\left(x, t_{m}\right)}\right)}{\theta\left(P_{\infty-}, D_{\hat{\mu}\left(x_{0}, t_{m, 0}\right)}\right) \theta\left(P_{\infty+}, D_{\hat{\mu}\left(x, t_{m}\right)}\right)}-2 e_{0,0}, \\
v\left(x, t_{m}\right)=-\frac{1}{w_{0}^{2}} \frac{\theta\left(P_{\infty-}, D_{\hat{v}\left(x, t_{m}\right)}\right) \theta\left(P_{\infty+}, D_{\hat{\mu}\left(x, t_{m}\right)}\right)}{\theta\left(P_{\infty+}, D_{\hat{v}\left(x, t_{m}\right)}\right) \theta\left(P_{\infty-}, D_{\hat{\mu}\left(x, t_{m}\right)}\right)}
\end{gathered}
$$

where $0 \leq m \leq 1$.

Proof. We start with the proof of the theta function representation (4.49) for $\psi_{1}$. Without loss of generality, analogous to the proof of Lemma 3, we only treat the special case of (3.9) when $\alpha_{0}=$ $1, \alpha_{k}=0,1 \leq k \leq n+1$, in the following. First, we temporarily assume that

$$
\mu_{j}\left(x, t_{m}\right) \neq \mu_{j^{\prime}}\left(x, t_{m}\right) \text { for } j \neq j^{\prime} \text { and }\left(x, t_{m}\right) \in \tilde{M}
$$

for appropriate $\tilde{M} \subseteq M$, and define the right-hand side of (4.49) to be $\tilde{\psi}_{1}$. In order to prove $\psi_{1}=\tilde{\psi}_{1}$, we investigate the local zeros and poles of $\psi_{1}$ defined by (4.7). From (3.20), (3.21), (4.2) and (4.4), 
we have

$$
\begin{gathered}
-2 v\left(x^{\prime}, t_{m}\right) \phi\left(P, x^{\prime}, t_{m}\right) \underset{P \rightarrow \hat{\mu}_{j}\left(x^{\prime}, t_{m}\right)}{=} \partial_{x^{\prime}} \ln \left(\lambda-\mu_{j}\left(x^{\prime}, t_{m}\right)\right)+O(1), \\
-B^{(m)}\left(\lambda, x_{0}, s\right) \phi\left(P, x_{0}, s\right) \underset{P \rightarrow \hat{\mu}_{j}\left(x_{0}, s\right)}{=} \partial_{s} \ln \left(\lambda-\mu_{j}\left(x_{0}, s\right)\right)+O(1) .
\end{gathered}
$$

Together with (4.7), this implies

$$
\psi_{1}\left(P, x, t_{m}, x_{0}, t_{m, 0}\right)= \begin{cases}\left(\lambda-\mu_{j}\left(x, t_{m}\right)\right) O(1) & \text { as } P \rightarrow \hat{\mu}_{j}\left(x, t_{m}\right) \neq \hat{\mu}_{j}\left(x_{0}, t_{m, 0}\right), \\ O(1) & \text { as } P \rightarrow \hat{\mu}_{j}\left(x, t_{m}\right)=\hat{\mu}_{j}\left(x_{0}, t_{m, 0}\right), \\ \left(\lambda-\mu_{j}\left(x_{0}, t_{m, 0}\right)\right)^{-1} O(1) & \text { as } P \rightarrow \hat{\mu}_{j}\left(x_{0}, t_{m, 0}\right) \neq \hat{\mu}_{j}\left(x, t_{m}\right)\end{cases}
$$

with $P=(\lambda, y) \in \mathscr{K}_{n},\left(x, t_{m}\right),\left(x_{0}, t_{m, 0}\right) \in \tilde{M}$, and $O(1) \neq 0$. Hence $\psi_{1}$ and $\tilde{\psi}_{1}$ have identical zeros and poles on $\mathscr{K}_{n} \backslash\left\{P_{\infty+}, P_{\infty_{-}}\right\}$, which are all simple by hypothesis (4.53). Next, we study the behavior of $\psi_{1}$ and $\tilde{\psi}_{1}$ near $P_{\infty \pm}$. By (4.35), (4.45), (4.46) and (4.49), we find that $\psi_{1}$ and $\tilde{\psi}_{1}$ have identical exponential behavior up to order $O(1)$ near $P_{\infty \pm}$. Thus, $\psi_{1}$ and $\tilde{\psi}_{1}$ share the same singularities and zeros. Then the Riemann-Roch-type uniqueness proves that $\psi_{1}=\tilde{\psi}_{1}$. Hence (4.49) holds subject to (4.53).

Substituting (4.40) into (4.47) and comparing with (4.26), one finds

$$
v=-\frac{1}{\omega_{0} N\left(x, t_{m}\right)} \cdot \frac{\theta\left(P_{\infty+}, D_{\hat{\mu}\left(x, t_{m}\right)}\right)}{\theta\left(P_{\infty+}, D_{\hat{v}\left(x, t_{m}\right)}\right)}, \quad N\left(x, t_{m}\right)=w_{0} \frac{\theta\left(P_{\infty-}, D_{\hat{\mu}\left(x, t_{m}\right)}\right)}{\theta\left(P_{\infty-}, D_{\hat{v}\left(x, t_{m}\right)}\right)} .
$$

Given $N\left(x, t_{m}\right)$, one can determine $\phi$ in (4.48) from (4.47) and $\psi_{2}$ in (4.50) from $\psi_{2}=-\phi \psi_{1}$. Re-examining the asymptotic behavior of $\psi_{1}$ near $P_{\infty}$ yields

$$
\psi_{1}\left(P, x, t_{m}, x_{0}, t_{m, 0}\right) \underset{\zeta \rightarrow 0}{=} \exp \left(\int_{x_{0}}^{x} u\left(x^{\prime}, t_{m}\right) d x^{\prime}+O(\zeta)\right) \exp \left(-\zeta^{-1}\left(x-x_{0}\right)-\zeta^{-m}\left(t_{m}-t_{m, 0}\right)+O(\zeta)\right) .
$$

A comparison of (4.55), (4.45), (4.46) and (4.49) proves (4.51).

Hence, we prove this theorem on $\tilde{M}$. The extension of all these results from $\tilde{M}$ to $M$ then follows by continuity of the Abel map and nonspecialty of $D_{\hat{\mu}\left(x, t_{m}\right)}$ on $M$.

Therefore, quasi-periodic solutions of coupled KdV type equations (1.1) and (1.2) are (4.51), (4.52) for $m=0,1$, respectively.

\section{Acknowledgments}

This work was supported by National Natural Science Foundation of China (Grants No. 11171312 and No. 11126308), Science and Technology Research Key Projects of the Education Department of Henan Province (Project No. 12A110023) and the Research Foundation of Henan University of Technology (Grant No. 2009BS041).

\section{References}

[1] R. Hirota and J. Satsuma, Soliton solutions of a coupled Korteweg-de Vries equation, Phys. Lett. A 85 (1981) 407-408. 
[2] R. Hirota and J. Satsuma, A coupled KdV equation is one case of the four-reduction of the KP hierarchy, J. Phys. Soc. Jpn. 51 (1982) 3390-3397.

[3] R. Dodd and A. P. Fordy, On the integrability of a system of a coupled KdV equation, Phys. Lett. A 89 (1982) 168-170.

[4] H. C. Hu and Q. P. Liu, New Darboux transformation for Hirota-Satsuma coupled KdV system, Chaos, Solitons \& Fractals 17 (2003) 921-928.

[5] D. Levi, A hierarchy of coupled Korteweg-de Vries equation, Phys. Lett. A 95 (1983) 7-10.

[6] W. H. Tam, W. X. Ma, X. B. Hu, D. L. Wang, The Hirota-Satsuma coupled KdV equation and a coupled Ito system revisited, J. Phys. Soc. Jpn. 69 (2000) 45-52.

[7] M. Ito, Symmetries and conservation laws of a coupled nonlinear wave equation, Phys. Lett. A 91 (1982) 335-338.

[8] B. Fuchssteiner, The Lie algebra structure of degenerate Hamiltonian and bi-Hamiltonian systems, Prog. Theor. Phys. 68 (1982) 1082-1104.

[9] D. Levi, A. Sym and S. Wojciechowski, A hierarchy of coupled KdV equations and normalisation conditions of the Hilbert-Riemann problem, J. Phys. A: Math. Gen. 16 (1983) 2423-2432.

[10] V. G. Drinfel'd and V. V. Sokolov, Lie algebras and equations of Korteweg-de Vries type, J. Math. Sci. 30 (1985) 1975-2036.

[11] Y. Nutku and Ö. Ogũz, Bi-Hamiltonian structure of a pair of coupled KdV equations, Nuovo Cimento B 105 (1990) 1381-1383.

[12] C. W. Cao and X. G. Geng, C Neumann and Bargmann systems associated with the coupled Kdv soliton hierarchy, J. Phys. A: Math. Gen. 23 (1990) 4117-4125.

[13] A. Yu. Zharkov, Computer classification of the integrable coupled KdV-like systems with unit main matrix, J. Symb. Comput. 15 (1993) 85-90.

[14] M. V. Foursov, On integrable coupled KdV-type systems, Inverse Problems 16 (2000) 259-274.

[15] X. M. Li and X. G. Geng, Lax matrix and a generalized coupled KdV hierarchy, Physica A 327 (2003) 357-370.

[16] F. Gesztesy and R. Ratnaseelan, An alternative approach to algebro-geometric solutions of the AKNS hierarchy, Rev. Math. Phys. 10 (1998) 345-391.

[17] F. Gesztesy and H. Holden, Soliton Equations and their Algebro-Geometric Solutions (Cambridge University Press, Cambridge, 2003).

[18] X. G. Geng, Algebraic-geometrical solutions of some multidimensional nonlinear evolution equations, J. Phys. A: Math. Gen. 36 (2003) 2289-2303.

[19] X. G. Geng and Bo Xue, Soliton solutions and quasiperiodic solutions of modified KortewegCde Vries type equations, J. Math. Phys. 51 (2010) 063516.

[20] X. G. Geng and Bo Xue, Quasi-periodic solutions of mixed AKNS equations, Nonlinear Analysis 73 (2010) 3662-3674.

[21] P. Griffiths and J. Harris, Principles of Algebraic Geometry (Wiley, New York, 1994).

[22] D. Mumford, Tata lectures on theta II (Birkhäuser, Boston, 1984). 